\title{
YEATS Domain
}

National Cancer Institute

\section{Source}

National Cancer Institute. YEATS Domain. NCI Thesaurus. Code C133715.

A protein domain whose structure is comprised of an immunoglobulin-like fold that is found in various chromatin modification factors and may bind to modified lysines in other proteins. 